\title{
Propagation effect of gravitational wave on detector response
}

\author{
Zhe Chang, Chao-Guang Huang, and Zhi-Chao Zhao \\ Institute of High Energy Physics and Theoretical Physics Center for Science Facilities, \\ Chinese Academy of Sciences, Beijing 100049, China
}

\begin{abstract}
The response of a detector to gravitational wave is a function of frequency. When the time a photon moving around in the Fabry-Perot cavities is the same order of the period of a gravitational wave, the phase-difference due to the gravitational wave should be an integral along the path. We present a formula description for detector response to gravitational wave with varied frequencies. The LIGO data for GW150914 and GW 151226 are reexamined in this framework. For GW150924, the traveling time of a photon in the LIGO detector is just a bit larger than a half period of the highest frequency of gravitational wave and the similar result is obtained with LIGO and Virgo collaborations. However, we are not always so luck. In the case of GW151226, the time of a photon traveling in the detector is larger than the period of the highest frequency of gravitational wave and the announced signal cannot match well the template with the initial black hole masses $14.2 \mathrm{M}_{\odot}$ and $7.5 \mathrm{M}_{\odot}$.
\end{abstract}

The detection of gravitational waves (GW) in the event GW150914 by the two advanced detectors of the Laser Interferometer Gravitational-wave Observatory (LIGO) [1] opens a new era for the direct detection of GW [2], searching black hole coalescence [3] and 'heavy' black holes with more than 25 solar mass [4], test of general relativity [5], understanding the astrophysical environment of black hole formation [6], etc. In one words, the era of multi-messenger astronomy has begun [1-9].

The analysis of GW150914 shows that the initial black hole masses are $36 \mathrm{M}_{\odot}$ and $29 \mathrm{M}_{\odot}$, which is heavier than the previous known stellar-mass black holes [10]. In the newly announced black hole merge event, GW151226 [11], the initial black hole masses are about $14 \mathrm{M}_{\odot}$ and $8 \mathrm{M}_{\odot}$, which fall into the known mass range of stellar black holes in the previous observations. The signals from noise for GW150914 and GW151226 are extracted by the same methods (for example, see $[12,13]$ ). It seems to make the observational results more reliable.

In this Letter, we try to provide a different method from Ref. [12] to determine the detector response to GW. Our basic observation is that a photon moves along null geodesics in Fabry-Perot cavities (except the reflections by the mirrors) no matter whether GW exists or not. We shall present a frequencydependent modulation for detector response to GW, and reexamine the GW150914 and GW151226 data carefully.

For simplicity, we consider a GW with frequency $f$ and polarization $h_{+}$incident normally to a detector, and assume the polarization along the detector's $x-$ $y$ arms. The lengths of the two cavities are supposed to be the same.

The metric of the linearized plane GW has the well-known form

$$
\mathrm{d} s^{2}=-\mathrm{d} t^{2}+\left(1+h_{11}\right) \mathrm{d} x^{2}+\left(1-h_{11}\right) \mathrm{d} y^{2}+\mathrm{d} z^{2} .
$$

The time-difference that a photon travels for a round trip along null geodesics in two Fabry-Perot cavities in a detector is

$$
\begin{aligned}
& \Delta t_{1}:= \frac{2}{c}\left(\oint\left(1+\frac{1}{2} h_{11}\right) \mathrm{d} x-\oint\left(1-\frac{1}{2} h_{11}\right) \mathrm{d} y\right) \\
&=\frac{1}{c}\left(\int_{0}^{L} h_{11} \mathrm{~d} x-\int_{L}^{0} h_{11} \mathrm{~d} x\right. \\
&\left.\quad \quad+\int_{0}^{L} h_{11} \mathrm{~d} y-\int_{L}^{0} h_{11} \mathrm{~d} y\right) \\
&=\left.\frac{4 L}{c} \frac{\sin (2 \pi f L / c)}{2 \pi f L / c} h_{11}(t+L / c)\right|_{z=0},
\end{aligned}
$$

where $L$ is the length of a Fabry-Perot cavity, $t$ is the initial time of the round trip, $z=0$ denotes the plane the detector lies on. It is the double of the timedifference in a same-size Michelson interferometer [12]. Since the frequency of GW observed is low enough $(f<700 \mathrm{~Hz})$, the inequality $2 f L / c \ll 1$ is always valid, and Eq.(2) reduces to

$$
\Delta t_{1}(t)=\left.\frac{4 L}{c} h_{11}(t+L / c)\right|_{z=0} .
$$

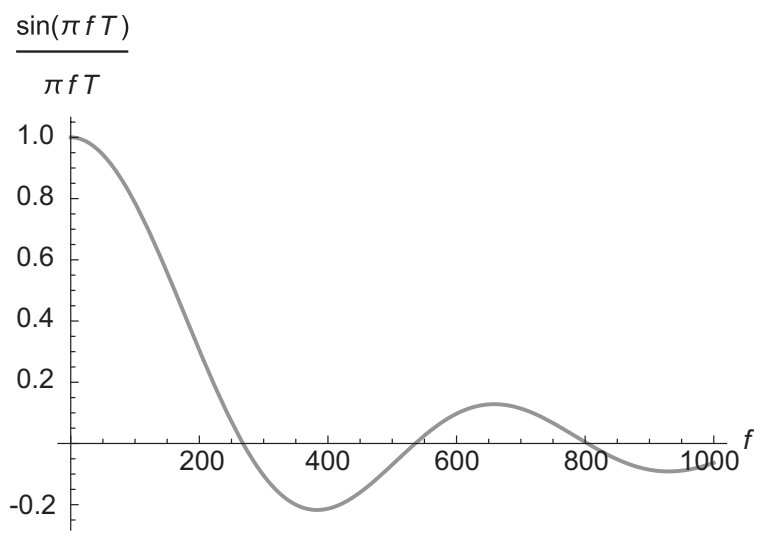

FIG. 1: Modulation function due to the propagation effect of GW. 

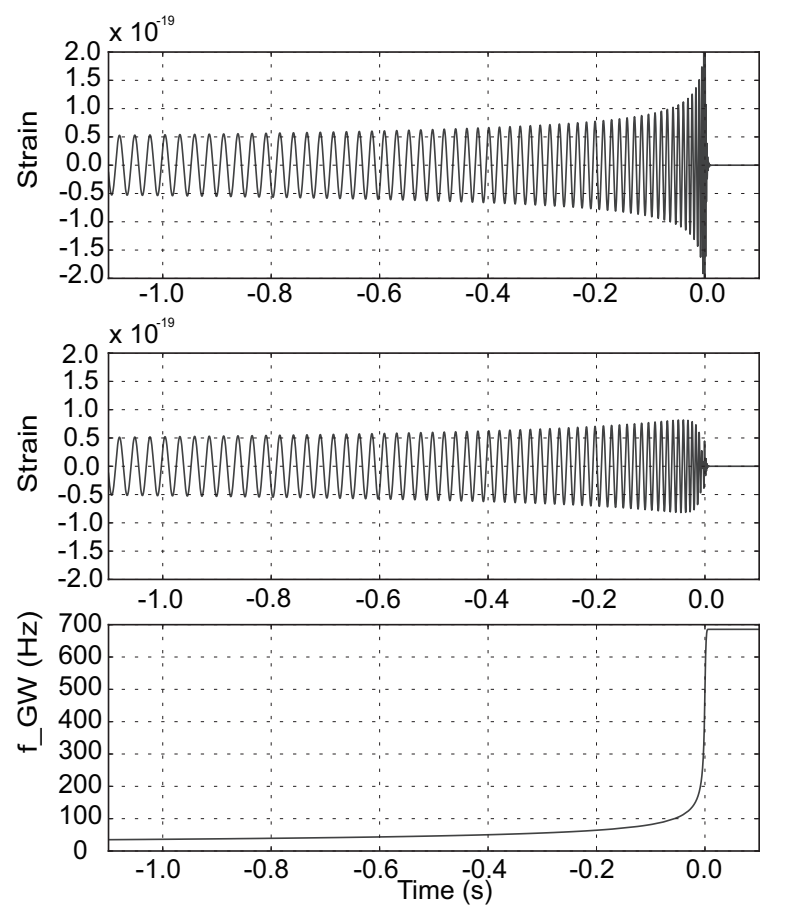

FIG. 2: The first panel: Strain of time of the template for plus polarization for GW151226 provided on LIGO website. The second panel: Strain of time of the same template with the propagation effect of GW being taken into account. The third panel: The varying frequency of GW with time.

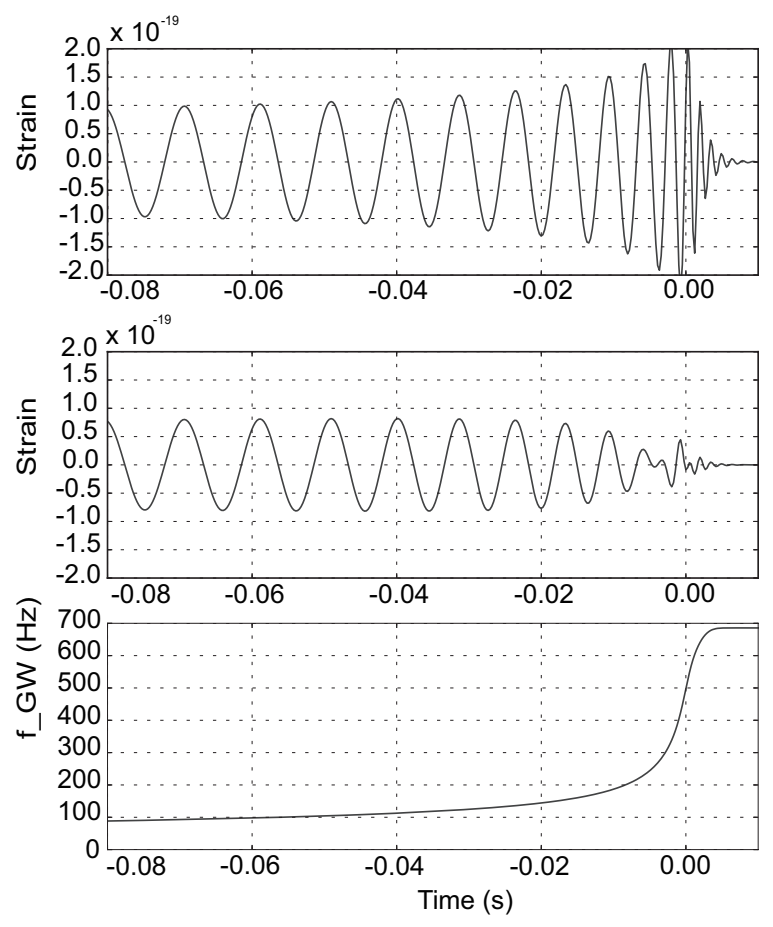

FIG. 3: The high-frequency part of FIG. 2.

In average, each photon will travel $\langle N\rangle \approx \mathcal{F} / \pi \approx$ 140 round trips in the cavity, where $\mathcal{F}$ is the finesse
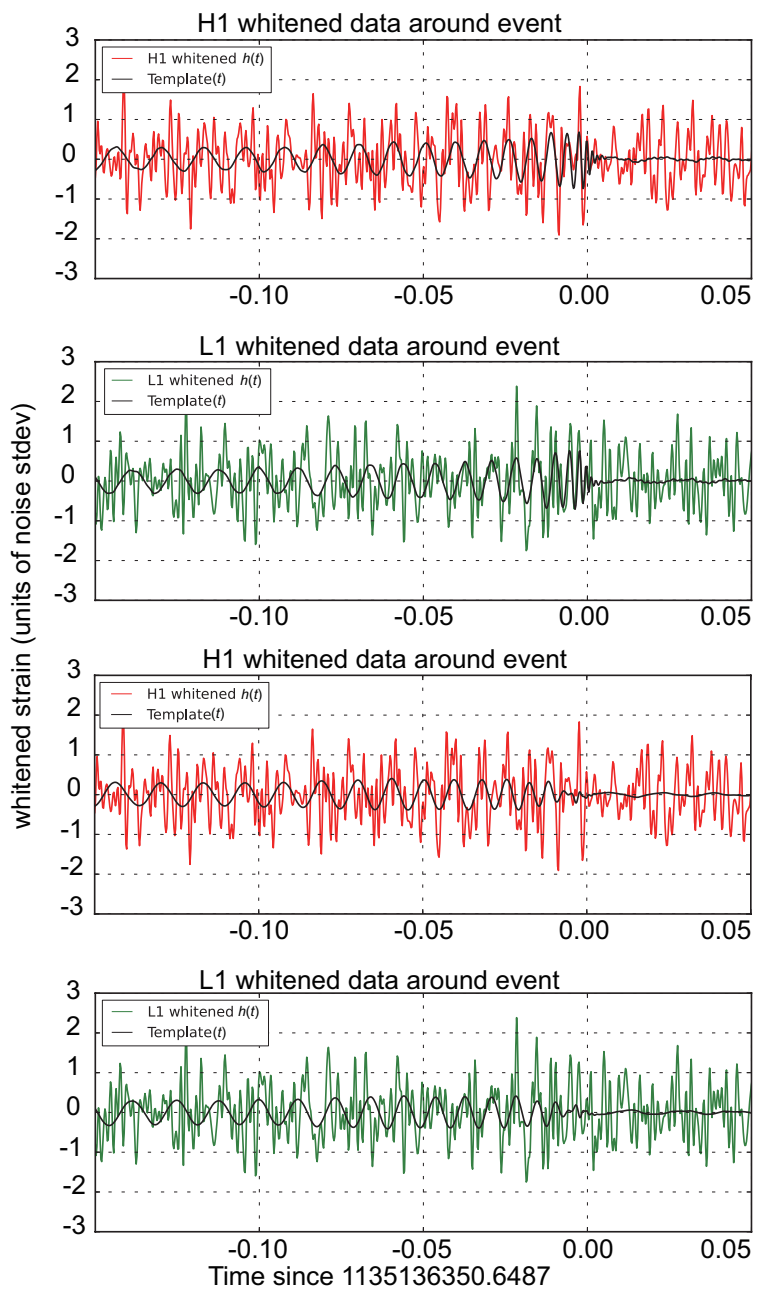

FIG. 4: The first and second panels show the matching of the GW signal GW151226 observed by LIGO and the template without taking into account the propagation effect of GW. The third and fourth panels show matching of the GW signal GW151226 observed by LIGO and template by taking into account the propagation effect of GW. The red and green curves denote GW strain projected onto each detector of the LIGO, and the black curve is the template.

of the cavities. Due to the propagation of GW, the gravitational field in which the photon travels in cavities are different time by time. Then, the time-difference that a photon travels for $\langle N\rangle$-round trip should be

$$
\begin{aligned}
\Delta t_{\langle N\rangle} & =\left.\frac{4 L}{c} \sum_{n=1}^{\langle N\rangle} h_{11}\right|_{z=0} \\
& =\left.\frac{4 L}{c} \frac{\mathcal{F}}{\pi} \frac{\sin (\pi f T)}{\pi f T} h_{11}(t+T / 2)\right|_{z=0},
\end{aligned}
$$

where $T=2 L\langle N\rangle / c$. The signal recorded by a detector is proportional to the time-difference. In the case of $2 L f\langle N\rangle / c \ll 1$, the wave length of GW 
is much longer than the $2\langle N\rangle$ times of cavity length,

$$
\left.\Delta t_{\langle N\rangle} \approx \frac{4 L}{c} \frac{\mathcal{F}}{\pi} h_{11}(t+T / 2)\right|_{z=0}
$$

The signal will be enhanced by $2 \mathcal{F} / \pi$ times compared with a same-size Michelson interferometer. However, in a generic case, the measured signal should have a suppression factor $\sin (\pi f T) /(\pi f T)$, which is a function of the frequency of GW. Figure 1 shows the propagation effect of GW on the signals measured by a detector. The effect makes the signal vanish when the photon's traveling time equals the inverse of GW frequency. From FIG. 1, one can find that even when the frequency of GW is about $150 \mathrm{~Hz}$ (at which the gravitational radiation is strongest in the event GW150914), the suppression factor should be about 0.558 .

In the following, we reexamine the GW150914 and GW151226 by taking into account the propagation effect of GW on the signals detected by the LIGO detectors. The program, the data, and the best fit template used here are all provided by LIGO$^{1}$. It should be noticed that the results obtained here will not match precisely with what released by the LIGO and Virgo collaborations, due to various subtleties in the analysis that are not addressed on LIGO website $^{1}$ and thus are ignored here. But these various subtleties are smaller than the propagation effect of GW considered here.

Figures 2 and 3 show the comparison between the template for GW151226 provided by LIGO ${ }^{1}$ and the one in which the propagation effect of GW has been taken into account. Figure 3 is the high-frequency part of FIG.2. It is easy to notice that for the lowfrequency of GW the propagation effect is small.

Figure 4 is the matching of GW signal and the templates. When the propagation effect of $\mathrm{GW}$ is ignored, the result is similar to LIGO and Virgo collaborations. It can be read out from first two panels of FIG. 4. The third and fourth panel show that, after the propagation effect of GW is taken into account, it is difficult to ensure the matching of the signal and template.

We also reexamined the data for GW150914. The results are presented in Figures 5, 6, and 7. Due to the frequency of the gravitational radiation in the event GW150914 is much lower than GW151226, the propagation effect for GW150914 is smaller than that for GW151226. Even so, there is still an observable propagation effect of GW in highfrequency part.

\footnotetext{
${ }^{1}$ https: \\losc.ligo.org \tutorials $\backslash$
}
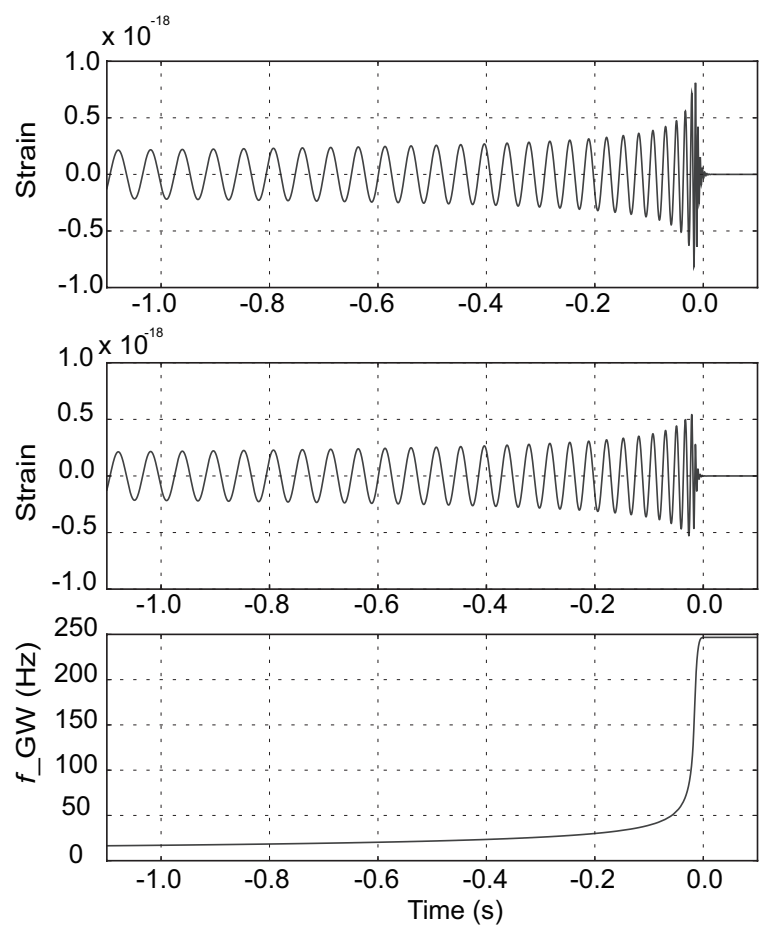

FIG. 5: The first panel: Strain of time of the template for plus polarization for GW150914 provided on LIGO website $^{1}$. The second panel: Strain of time of the same template with the propagation effect of GW being taken into account. The third panel: The varying frequency of GW with time.
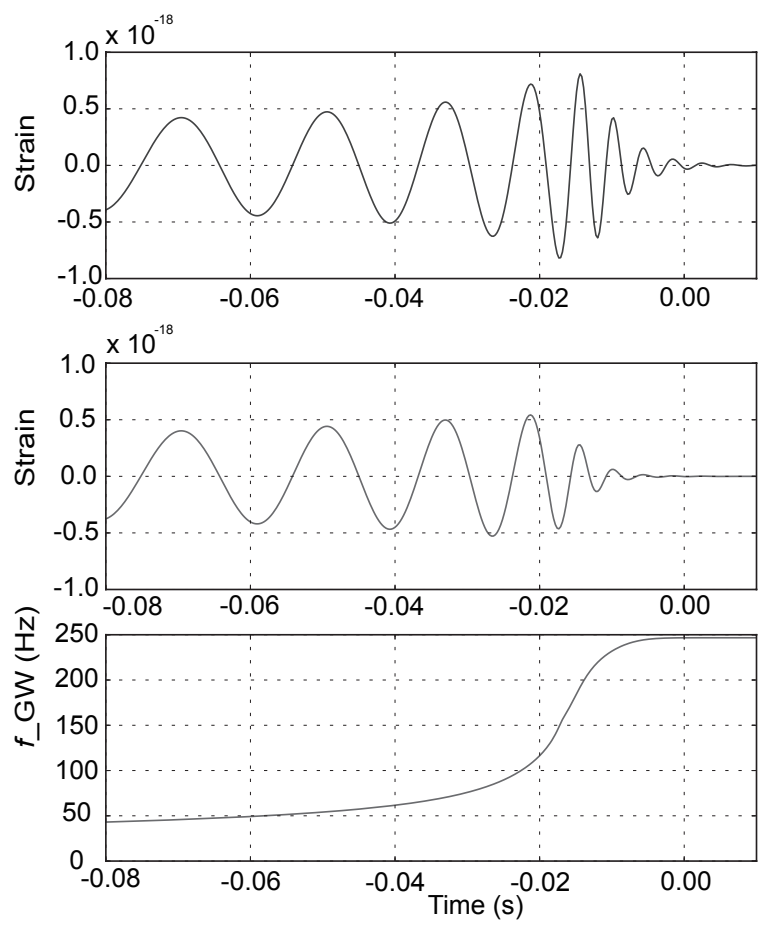

FIG. 6: The high-frequency part of FIG.5. 

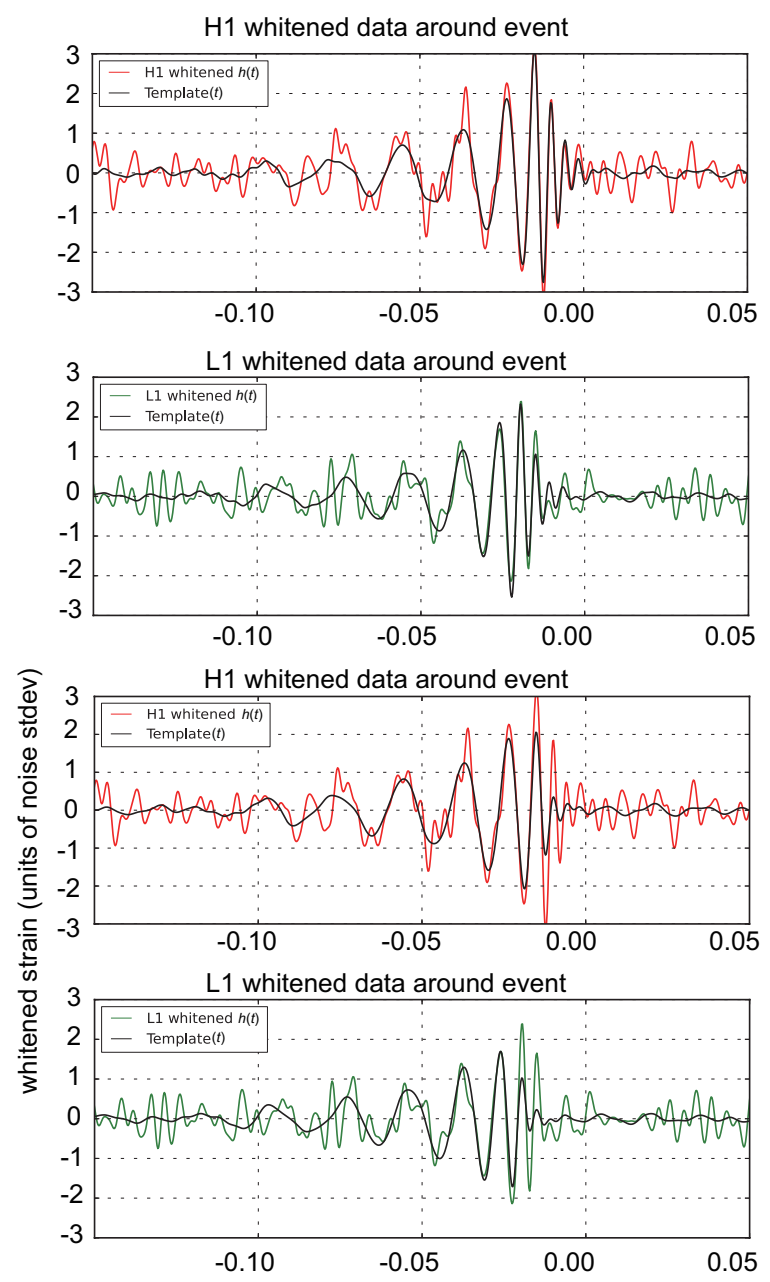

FIG. 7: The first and second panels show the matching of the GW signal GW150914 observed by LIGO and the template without taking into account the propagation effect of GW. The third and fourth panels show matching of the GW signal GW150914 observed by LIGO and template by taken into account of the propagation effect of GW. The red and green curves denote GW strain projected onto each detector of the LIGO, and the black curve is the template.

As a conclusion, the propagation effect for highfrequency GW is important in matching the signals observed with templates. Although our analysis disfavors GW151226, it is still possible to find more reliable GW signals from observation data according to the new templates after the propagation effect is taken into account. Finally, it should be remarked that the modulation function for detector response, $\frac{\sin (\pi f T)}{\pi f T}$, is calculated from GW with a fixed frequency and amplitude. The frequency and amplitude of the actual GW signals vary time by time. So, the quantitative analysis should be an integral of the time-difference of a photon along the paths traveling in the two cavities.

\section{Acknowledgment}

We would like to thank Profs. Yong-Gui Li, ZhouJian Cao, and Xi-Long Fan for helpful discussion. This work is supported by National Natural Science Foundation of China under the grants 11275207 and 11375203 .

[1] B. P. Abbott, et al, Phys. Rev. Lett. 116061102 (2016)

[2] B. P. Abbott, et al, Phys. Rev. Lett. 116131103 (2016)

[3] B. P. Abbott, et al, Phys. Rev. D 93122003 (2016)

[4] B. P. Abbott, et al, Phys. Rev. Lett. 116241102 (2016)

[5] B. P. Abbott, et al, Phys. Rev. Lett. 116221101 (2016)

[6] B. P. Abbott, et al, Ap.J. Lett. 818 L22 (2016)

[7] D. Blair, et al, Sci. Chin. Phys. Mech. Astron. 58, $120402(2015)$

[8] H. M. Lee, et al, Sci. Chin. Phys. Mech. Astron. 58, 120405 (2015)

[9] X. L. Fan, Sci. China-Phys. Mech. Astron. 59, $640001(2016)$

[10] J. A. Orosz, et al, Nature 449872 (2007)

[11] B. P. Abbott, et al, Phys. Rev. Lett. 116241103 (2016)

[12] E. D. Black and R. N. Gutenkunst, Am. J. Phys. 71 365 (2003)

[13] B. Allen, W. G. Anderson, P. R. Brady, D. A. Brown, and J. D. E. Creighton, Phys. Rev. D 85 122006 (2012) 